\title{
Melatonin sensitizes esophageal cancer cells to 5-fluorouracil via promotion of apoptosis by regulating EZH2 expression
}

\author{
MENGTI ZHANG ${ }^{1}$, MENGLI ZHANG $^{2}$, RUIJIA LI $^{3}$, RUI ZHANG $^{4}$ and YUELI ZHANG ${ }^{1}$ \\ ${ }^{1}$ Department of Clinical Pharmacy, Zhengzhou Central Hospital Affiliated to Zhengzhou University, Zhengzhou, \\ Henan 450007; ${ }^{2}$ Department of Traditional Chinese Medicine, Kaifeng Central Hospital, Kaifeng, Henan 475000; \\ ${ }^{3}$ Department of Pharmacy, The 8th Hospital of Xi'an; ${ }^{4}$ Department of Critical Care Medicine, \\ Shaanxi Provincial Cancer Hospital, College of Medicine, Xi'an Jiaotong University, Xian, Shaanxi 710061, P.R. China
}

Received August 15, 2020; Accepted January 13, 2021

DOI: $10.3892 /$ or.2021.7973

\begin{abstract}
The present study aimed to investigate the effects of melatonin (MLT) and 5-fluorouracil (5-FU) combination on the chemotherapeutic effect of 5-FU in esophageal cancer, and determine the potential molecular mechanisms. The effects of MLT and 5-FU combination on cell proliferation, cell migration and invasion, and cell apoptosis were detected by Cell Counting Kit-8, Transwell assays and flow cytometric analysis, respectively. Quantitative PCR and western blotting were performed for mRNA and protein quantification, respectively. The present study revealed that MLT significantly inhibited cell activity in a dose-dependent manner and MLT significantly enhanced 5-FU-mediated inhibition of cell proliferation in esophageal cancer cells. Compared with the 5-FU group, the MLT and 5-FU combination group significantly inhibited the invasion and migration of EC-9706 and EC-109 cells. The present study also revealed that MLT and 5-FU synergistically promoted apoptosis via activation of the caspase-dependent apoptosis pathway. Histone-lysine N-methyltransferase EZH2 (EZH2) was highly expressed in esophageal cancer tissues and cells and its high expression promoted esophageal cancer progression. MLT and 5-FU combination inhibited cell proliferation and promoted apoptosis by regulating EZH2 expression. In conclusion, MLT enhanced 5-FU-mediated inhibition of cell proliferation via promotion of apoptosis by regulating EZH2 expression in esophageal cancer.
\end{abstract}

\section{Introduction}

Esophageal cancer (EC) is the eighth most common cancer and the sixth most common cause of cancer-related deaths

Correspondence to: Dr Yueli Zhang, Department of Clinical Pharmacy, Zhengzhou Central Hospital Affiliated to Zhengzhou University, 195 Tongbai Road, Zhengzhou, Henan 450007, P.R. China E-mail: zhangyueli0228@163.com

Key words: melatonin, 5-fluorouracil, esophageal cancer, histone-lysine $\mathrm{N}$-methyltransferase EZH2, apoptosis worldwide $(1,2)$. Radical surgery is the preferred treatment for EC, however $80 \%$ of patients with EC are unable to undergo radical surgery by the time of diagnosis (3). Therefore, radiotherapy or chemotherapy maintenance is the main treatment for patients with advanced EC (4). Despite the rapid and ongoing development of chemotherapeutic drug development and multimodal therapies that have facilitated treatment for patients with EC, the prognoses and outcomes typically remain unsatisfactory. High incidences of chemotherapeutic drug resistance have become one of the important causes of failure in the treatment of EC (5). Therefore, it is urgent and necessary to develop innovative treatment strategies for EC.

5-Fluorouracil (5-FU) is one of the most common chemotherapeutic drugs used in the treatment of EC (6). 5-FU inhibits thymidylate synthase and interferes with DNA synthesis through its metabolite FdUMP. In addition, its metabolite FdUTP can affect cell metabolism in numerous manners, such as transcription, translation and post-translation modification through the incorrect addition into the RNA (7). 5-FU is characterized by a narrow therapeutic index due to its toxicity to normal cells, and cancer cells are easily resistant to 5-FU. These two factors are the main obstacles to the clinical application of 5-FU (8). Hence, there is an urgent need to discover other drug reagents to be used in combination with 5-FU to improve the sensitivity of cancer cells to 5-FU and reduce its toxicity to normal cells.

Melatonin (MLT) is an amine hormone produced in the conarium of mammals and humans (9). In recent years, studies have revealed that MLT has a number of physiological functions, such as promoting sleep, regulating jet lag, anti-aging effects, regulating immunity and antitumor effects (10-13). Further biological function studies have revealed that MLT can regulate the proliferation, cycle and apoptosis of cancer cells (14-16). MLT increases the efficacy of chemotherapeutic drugs by increasing its sensitivity of chemotherapy drugs, reducing the probability of drug resistance, and reducing the toxicity to normal cells (17-20). However, the mechanisms and dynamics of MLT and its effects on the chemotherapeutic effect of 5-FU in EC have rarely been examined and reported.

Methyl-transferase EZH2 is a component of polycomb repressive complex 2 . Accumulating studies have indicated that EZH2 is upregulated in several types of human cancers (21). 
Our previous research also revealed that EZH2 was upregulated in samples afflicted by EC and EZH2 was confirmed as an oncogene in esophageal cancer via biological experiments (22). Recent studies have revealed that EZH2 promoted chemotherapeutic drug resistance in numerous cancers $(23,24)$. In addition, previous studies also revealed that melatonin inhibited the tumorigenicity of glioblastoma stem-like cells via the EZH2 signaling axis $(25,26)$. Hence, it was hypothesized that MLT and 5-FU combination inhibits cell proliferation and promotes apoptosis by regulating the expression of $\mathrm{EZH} 2$.

The present study sought to examine whether or not MLT affects the chemotherapeutic effects of 5-FU, namely via its ability to increase the sensitivity of EC cells to 5-FU and its subsequent downstream effects. It was hypothesized that MLT may play a role in sensitizing or synergizing EC cellular response to 5-FU treatment. EC cells were treated with MLT and 5-FU alone or in combination and the effects of MLT and 5-FU combination on cell proliferation, migration, invasion and apoptosis in EC-9706 and EC-109 cells were analyzed. The present study also observed the effects of MLT and 5-FU co-treatment on EZH2 expression to determine the potential molecular mechanisms.

\section{Materials and methods}

Cell culture and transfection. Human EC cell lines (EC-9706 and EC-109) and an immortalized normal esophagus epithelial cell line (HET-1A) were obtained from The Cell Bank of the Chinese Academy of Sciences (Shanghai, China). Cell were cultured in RPMI-1640 medium supplemented with 10\% FBS (Gibco; Thermo Fisher Scientific, Inc.) and $100 \mathrm{U} / \mathrm{ml}$ of penicillin and streptomycin (Gibco; Thermo Fisher Scientific, Inc.). Cells were incubated in a humidified atmosphere at $37^{\circ} \mathrm{C}$ and $5 \% \mathrm{CO}_{2}$. Cells were transfected with the following small interfering RNAs (siRNAs; transfection concentration $2 \mu \mathrm{g} / \mathrm{ml}$ ): EZH2-specific si-RNA (si-EZH2-1, 5'-AAGACTCTGAAT GCAGTTGCT-3'; si-EZH2-2, 5'-GGAUGGUACUUUCAU UGAATT-3'), siRNA negative control (si-NC, 5'-UUCUCC GAACGUGUCACGUTT-3') (all from Shanghai GenePharma Co., Ltd.). si-EZH2-1 (hereafter referred to as si-EZH2) was used for further experiments. The pcDNA3.1 empty vector (pcDNA-NC) and pcDNA3.1-EZH2 overexpression vector (pcDNA-EZH2; transfection concentration $2 \mu \mathrm{g} / \mathrm{ml}$ ) were designed and produced by Shanghai GeneChem Co., Ltd.

Lipofectamine ${ }^{\circledR} 2000$ (Invitrogen; Thermo Fisher Scientific, Inc.) was used to transfect cells with si-RNA and pcDNA-EZH2 according to the manufacturer's protocols. Cells were incubated at $37^{\circ} \mathrm{C}$ and $5 \% \mathrm{CO}_{2}$, for $48 \mathrm{~h}$ for RT-qPCR and $72 \mathrm{~h}$ for western blotting. All transfection experiments were repeated three times. The transfection efficiency was confirmed by reverse transcription-quantitative PCR (RT-qPCR) analysis.

EZH2 expression in EC from the cancer genome atlas (TCGA) database. EZH2 expression in EC was extracted from the public database starBase 3.0 online tool (http://starbase.sysu. edu.cn/) (27).

RNA extraction and reverse transcription $R T-q P C R$. TRIzol ${ }^{\circledR}$ (Invitrogen; Thermo Fisher Scientific, Inc.) was used to extract total RNA from cells. Subsequently, samples were treated with DNase I and total RNA was quantified using a NanoDrop 2000. Samples were reverse transcribed into cDNA. A total of $20 \mu \mathrm{l}$ qPCR reaction mixture was run on an ABI 7500 Fast RT-qPCR system for qPCR. GAPDH was used as the internal control. The following primer pairs were used for the qPCR: EZH2 forward, 5'-AGGACGGCTCCTCTAACCAT-3' and reverse, 5'-CTTGGTGTTGCACTGTGCTT-3'; and GAPDH forward, 5'-GGGAGCCAAAAGGGTCAT-3' and reverse, 5'-GAGTCC TTCCACGATACCAA-3'. Relative expression levels were calculated using the $2^{-\triangle \Delta C q}$ method (28). Detailed steps were previously described in a published study (29).

Cell proliferation assay. Cell proliferation assays were performed using Cell Counting Kit-8 (CCK-8; Beyotime Institute of Biotechnology) reagent. Briefly, $5 \times 10^{3}$ cells were seeded into 96-well plates with $100 \mu \mathrm{l}$ of medium. Cells were incubated overnight at $37^{\circ} \mathrm{C}$. Upon reaching $70-80 \%$ confluence, fresh medium containing $0,0.125,0.25,0.5,1$ and $2 \mathrm{mM}$ melatonin (Sigma-Aldrich; Merck KGaA) or 0, 10, 20, 40, 60 and $80 \mathrm{mM}$ 5-FU (Sigma-Aldrich; Merck KGaA) were added. The cells were further incubated for $2 \mathrm{~h}$ with $10 \mu \mathrm{l}$ of CCK-8 solution per well and for another 24, 48, 72 and $96 \mathrm{~h}$. The absorbance (optical density) was measured at a wavelength of $450 \mathrm{~nm}$ using a Tecan microplate reader (Tecan Group, Ltd.).

Cell migration and invasion assay. Cell migration and invasion were assessed using Transwell assays. EC-9706 cells were treated with $1 \mathrm{mM}$ melatonin and $40 \mathrm{mM} 5-\mathrm{FU}$ and EC-109 cells was treated with $1 \mathrm{mM}$ melatonin and $60 \mathrm{mM}$ $5-\mathrm{FU}$, then harvested and resuspended in serum-free medium $\left(5 \times 10^{5}\right.$ cells $\left./ \mathrm{ml}\right)$. For the Transwell migration assays, $5 \times 10^{4}$ cells were seeded in the upper chamber. For the invasion assays, Transwell inserts were pre-coated with Matrigel solution and polymerized in the upper chamber. The upper chamber was filled with FBS-free medium and the lower chamber was filled with $600 \mu \mathrm{l}$ medium with $10 \%$ FBS. Cells were incubated for 24 at $37^{\circ} \mathrm{C}$. Cells that had migrated or invaded to the lower surface of the membrane were fixed for $20 \mathrm{~min}$ with $4 \%(\mathrm{v} / \mathrm{v})$ paraformaldehyde, stained with $0.1 \%$ crystal violet (Sigma-Aldrich; Merck KGaA) for $20 \mathrm{~min}$, and counted from five independent visual fields by an inverted microscope (magnification, x100; Olympus Corp.).

Cell apoptosis assay. Cell apoptosis assays were performed using an Annexin V/PI double-staining assay (Sigma-Aldrich; Merck KGaA). In brief, after EC-9706 cells were treated with $1 \mathrm{mM}$ melatonin and $40 \mathrm{mM} 5-\mathrm{FU}$ and EC-109 cells were treated with $1 \mathrm{mM}$ melatonin and $60 \mathrm{mM}$-FU for $24 \mathrm{~h}$, the cells $\left(2 \times 10^{5}\right.$ cells/well) were harvested and washed with PBS. Then, the cells were resuspended with $400 \mu 1 \mathrm{lX}$ binding buffer, followed by the addition of $5 \mu \mathrm{l}$ Annexin V-FITC and incubation at room temperature for $15 \mathrm{~min}$ and the addition and incubation of $10 \mu \mathrm{l} \mathrm{PI}$ on an ice bath for $5 \mathrm{~min}$. Cells were then analyzed using a flow cytometer (Beckman Coulter, Inc.). Data were analyzed using CytExpert software (version 2.3; Beckman Coulter, Inc.).

Western blotting. Proteins were extracted from EC cells using RIPA lysis buffer (Beyotime Institute of Biotechnology) 

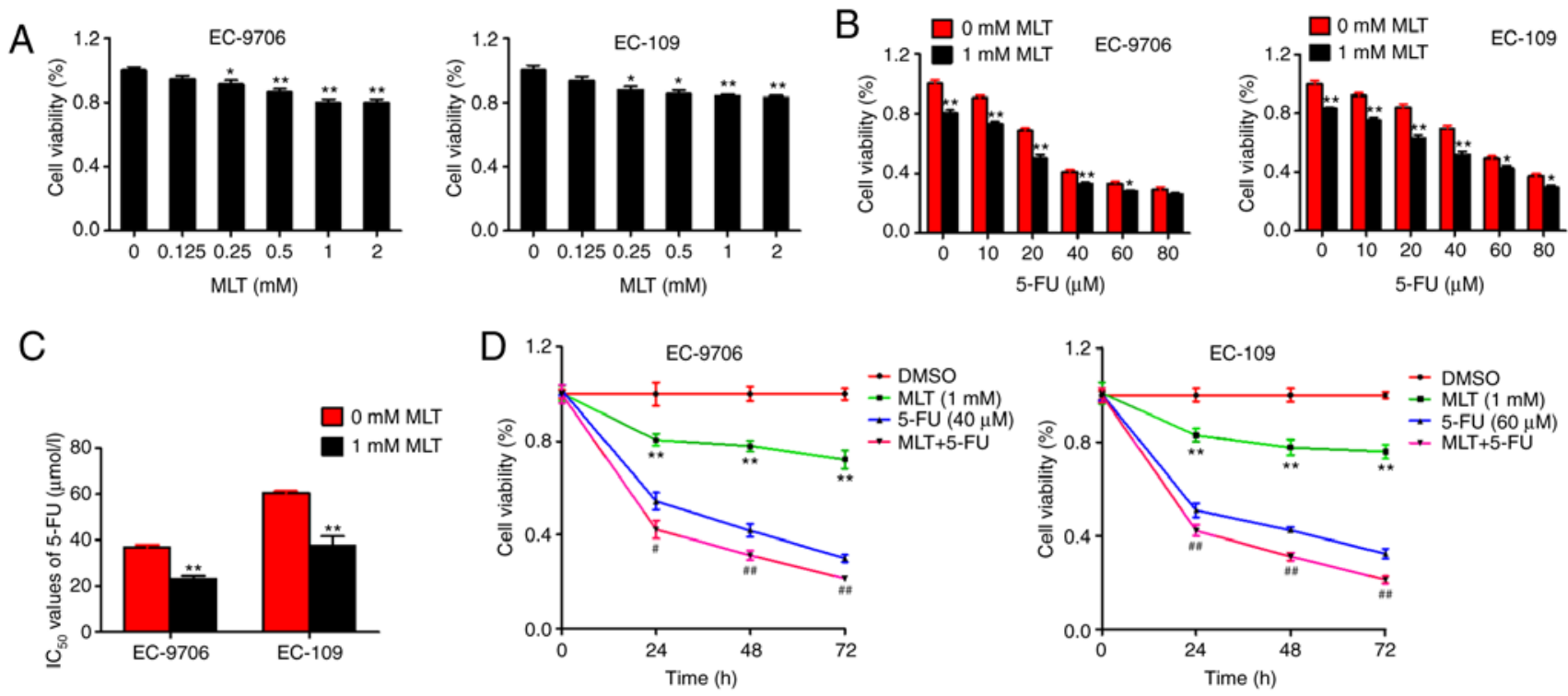

Figure 1. MLT and 5-FU combination enhances the inhibition of cell proliferation. (A) Changes in cell viability after EC-9706 and EC-109 cells were incubated with MLT for $24 \mathrm{~h}$. (B) Changes in cell viability after EC-9706 and EC-109 cells were incubated with 5-FU alone or in combination with MLT for $24 \mathrm{~h}$. (C) The $\mathrm{IC}_{50}$ values of 5-FU for the inhibition of cell viability in cells treated with or without MLT were determined. ${ }^{*} \mathrm{P}<0.05$ and ${ }^{* *} \mathrm{P}<0.01$ vs. the 0 -mM MLT group. (D) EC-9706 and EC-109 cells were incubated with 5-FU (40 or $60 \mu \mathrm{M}$, respectively) and MLT (1 mM) for 24,48 and 72 h. ${ }^{* *} \mathrm{P}<0.01$ vs. the DMSO group; ${ }^{\#} \mathrm{P}<0.05$ and ${ }^{\# \#} \mathrm{P}<0.01$ vs. the 5-FU group. MLT, melatonin; 5-FU, 5-fluorouracil.

containing protease $\mathrm{K}$ inhibitor. The protein concentration was determined with a BCA protein assay kit (Beijing Solarbio Life Sciences). Proteins (30 $\mu \mathrm{g} /$ lane) were separated via $12 \%$ SDS-PAGE and transferred onto PVDF membranes. Subsequently, the membranes were blocked with 5\% skim milk for $2 \mathrm{~h}$ at room temperature and washed three times with PBS. Samples were incubated at $4^{\circ} \mathrm{C}$ overnight with the following primary antibodies: Anti-Bcl-2 (1:500; product code ab32124), anti-Mcl-1 (1:500; product code ab32087), and anti-caspase-3 (1:500; product code ab32351; all from Abcam). Membranes were then incubated $2 \mathrm{~h}$ at room temperature with horseradish peroxidase-conjugated immunoglobulin $\mathrm{G}$ secondary antibody $(1: 2,000$; product code ab205718, Abcam). GAPDH (1:1,000; product code ab181602; Abcam) was used as an internal control gene. Protein bands were visualized using an ECL kit (Beyotime Institute of Biotechnology). A Chemidoc EQ system (Bio-Rad Laboratories, Inc.) was used to quantify western blot results. The densitometry of protein signals was analyzed with Quantity One software (version 4.62; Bio-Rad Laboratories, Inc.).

Statistical analysis. Statistical analyses were conducted using SPSS 19.0 software (IBM Corp.) and illustrated using GraphPad Prism 5.0 (GraphPad Software, Inc.). Comparisons between two groups were performed using unpaired Student's t-test, Comparisons of differences among $\geq 3$ groups were performed using one-way ANOVA followed by Tukey's test. $\mathrm{P}<0.05$ was considered to indicate a statistically significant difference.

\section{Results}

MLT and 5-FU combination enhances the inhibition of cell proliferation. The present study first observed the effects of MLT on cell proliferation in EC-9706 and EC-109 cells.
MLT (0-2 $\mathrm{mM})$ produced significant inhibition of cell activity in a dose-dependent manner in EC-9706 and EC-109 cells (Fig. 1A). Subsequently, to determine whether MLT enhances 5-FU-mediated inhibition of cell proliferation in EC-9706 and EC-109 cells, cells were treated with various concentrations of 5-FU alone or in combination with MLT. 5-FU alone inhibited cell activity in a dose-dependent manner. However, compared with 5-FU alone, the MLT and 5-FU combination significantly enhanced 5-FU-mediated inhibition of cell activity in EC-9706 and EC-109 cells (Fig. 1B).

Next, the present study analyzed the effects of MLT and 5 -FU combination treatment on the $\mathrm{IC}_{50}$ values of 5 -FU in EC-9706 and EC-109 cells. Compared with 5-FU alone, the $\mathrm{IC}_{50}$ values of MLT and 5-FU combination were significantly decreased (38.14 and 37.76 in EC-9706 and EC-109 cells, respectively) (Fig. 1C). In addition, the inhibition of MLT and 5-FU on cell activity occurred in a time-dependent manner either alone or in combination (Fig. 1D). These results suggested that MLT may enhance the chemotherapeutic effects of 5-FU in EC.

Effects of MLT and 5-FU combination on cell migration, invasion and apoptosis. To further investigate the effects of MLT and 5-FU on the biological function in EC, the present study first evaluated the effects of MLT and 5-FU on cell invasion and migration using Transwell assays. The results revealed that MLT treatment significantly inhibited invasion and migration of EC-9706 and EC-109 cells compared with the DMSO group. Similarly, compared with 5-FU alone, the MLT and 5-FU combination significantly inhibited invasion and migration of EC-9706 and EC-109 cells (Fig. 2A and B). These results indicated that MLT enhanced 5-FU-mediated inhibition of cell invasion and migration. Subsequently, the effects of MLT and 5-FU on cell apoptosis were assessed by flow cytometric analysis. The results revealed that MLT 
A

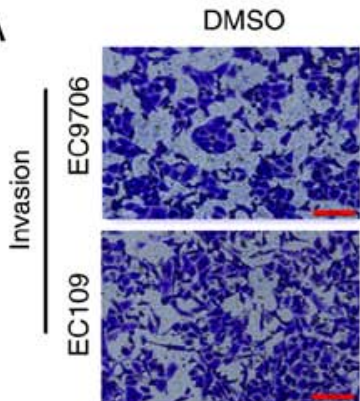

B

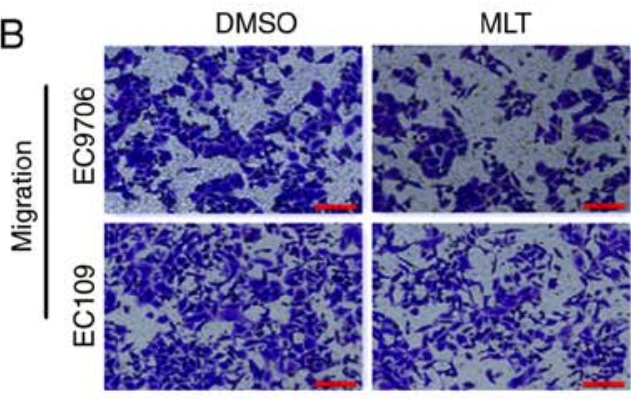

C

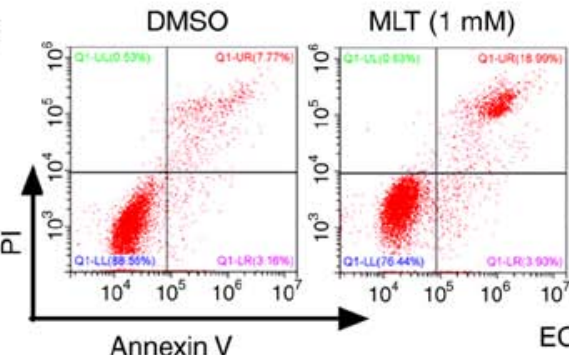

EC-9706

D

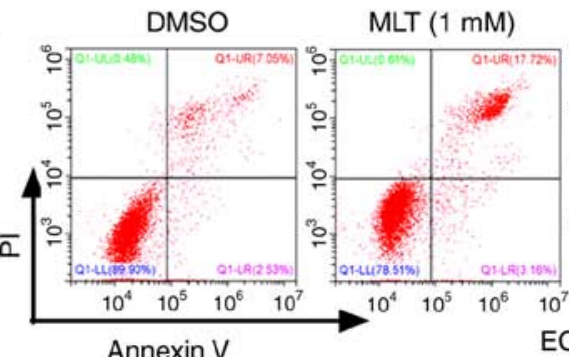

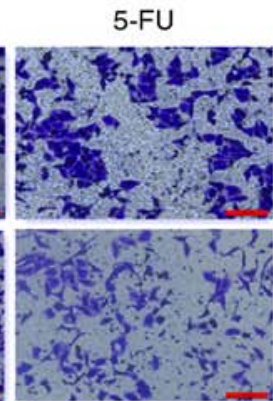

$5-\mathrm{FU}$

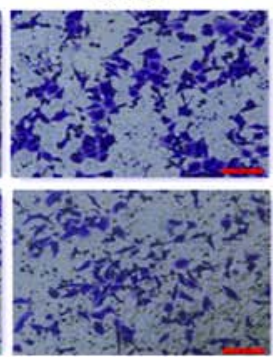

$5-\mathrm{FU}+\mathrm{MLT}$
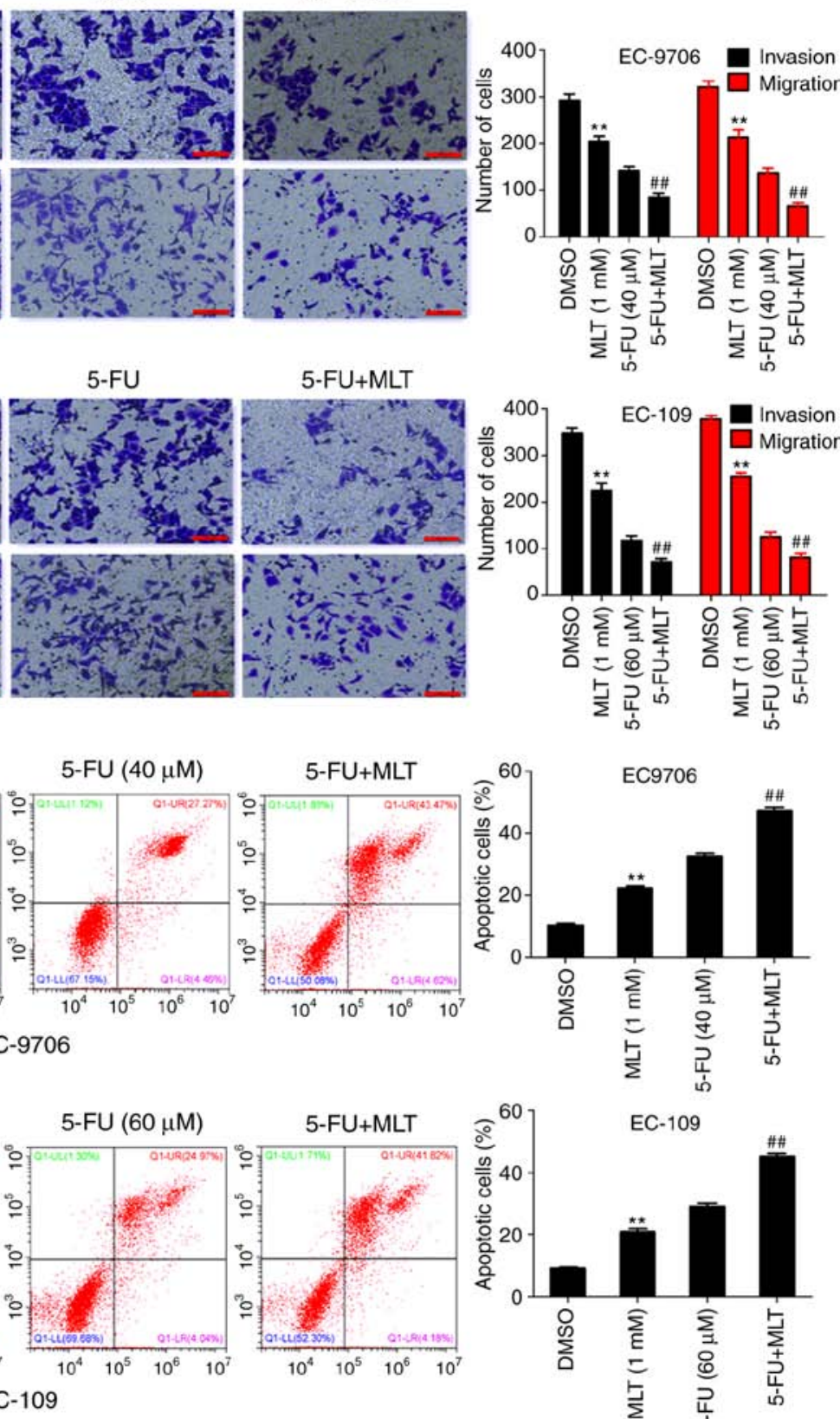

Figure 2. Effects of MLT and 5-FU combination on cell migration, invasion and apoptosis. (A and B) Effects of MLT and 5-FU combination on cell invasion and migration in EC-9706 and EC-109 cells (Scale bars, $100 \mu \mathrm{m})$. (C and D) Effects of MLT and 5-FU combination on EC-9706 and EC-109 cell apoptosis. ${ }^{* *} \mathrm{P}<0.01$ vs. the DMSO group; ${ }^{\# \#} \mathrm{P}<0.01$ vs. the 5-FU group. MLT, melatonin; 5-FU, 5-fluorouracil.

treatment significantly promoted cell apoptosis of EC-9706 and EC-109 cells compared with the DMSO group. Similarly, compared with 5-FU alone, the MLT and 5-FU combination significantly promoted apoptosis of EC-9706 and EC-109 cells (Fig. 2C and D). These results suggested that MLT and 5-FU synergistically promoted apoptosis in EC.

Role of EZH2 in EC. To investigate the expression levels of EZH2 in EC, the present study first extracted the expressional data of EZH2 in EC from TCGA database. The results revealed that the levels of EZH2 expression assessed in tumor tissues was higher compared with normal tissues (Fig. 3A). Subsequently, the levels of EZH2 expression were assessed in EC cell lines (EC-9706 and EC-109), and in a normal cell line (HET-1A). Both EC cell lines exhibited significantly higher levels of EZH2 expression compared with HET-1A cells (Fig. 3B). To further investigate the roles of EZH2 in EC, two EZH2 siRNAs were designed to knockdown EZH2. RT-qPCR and western blotting results revealed that EZH2 expression was significantly decreased in EC-9706 and EC-109 cells transfected with si-EZH2 (Fig. 3C and D). Next, the present study further investigated the effects of silencing EZH2 expression on the biological function in EC. CCK-8 assays revealed that EZH2 knockdown significantly inhibited cell proliferation of EC-9706 and EC-109 cells (Fig. 4A). Transwell assays revealed that EZH2 knockdown significantly inhibited cell invasion and migration of EC-9706 and EC-109 cells (Fig. 4B and C). However, silencing of EZH2 expression 
$A \widehat{\bar{\delta}}$ Data source: starBase v3.0 project
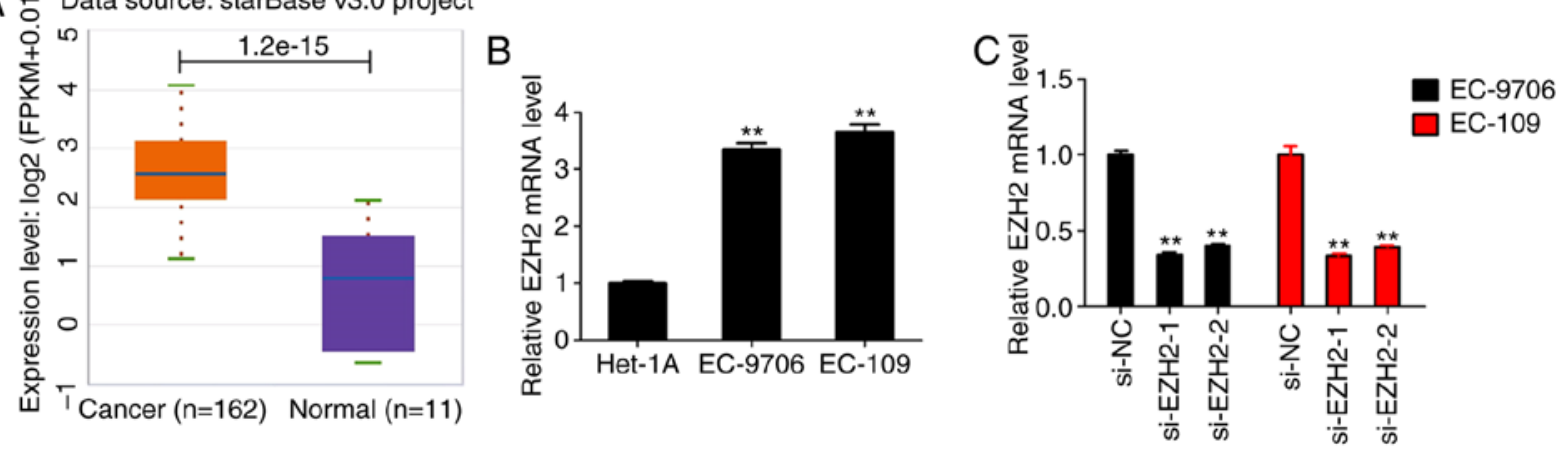

D
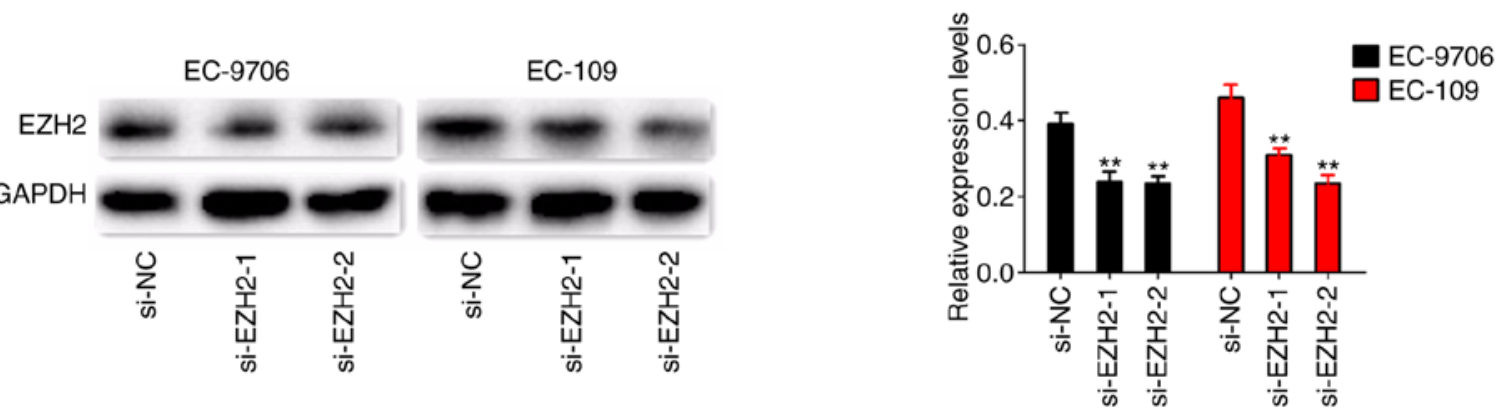

Figure 3. EZH2 expression in EC. (A) EZH2 expression in The Cancer Genome Atlas database. (B) EZH2 expression in EC cell lines and HET-1A cells. ${ }^{* *} \mathrm{P}<0.01$ vs. the HET-1A group. (C and D) EZH2 expression in EC-9706 and EC-109 cells transfected with si-EZH2 or si-NC. ${ }^{* *} \mathrm{P}<0.01$ vs. the si-NC group. EZH2, histone-lysine N-methyltransferase EZH2; EC, esophageal cancer; si, small interfering RNA; NC, negative control.

significantly promoted apoptosis in EC-9706 and EC-109 cells (Fig. 4D). These results suggested that EZH2 may act as an oncogene in EC.

MLT and 5-FU combination suppresses EZH2 expression. To further confirm the promotion of apoptosis in EC by MLT and 5-FU, protein expression levels were determined using western blotting. The results indicated that MLT and 5-FU co-treatment significantly inhibited the protein expression of Bcl-2 and Mcl-1, but enhanced cleaved caspase-3 expression in EC-9706 and EC-109 cells (Fig. 5A and B). Subsequently, the effects of MLT and 5-FU co-treatment on EZH2 expression were assessed. The results revealed that MLT and 5-FU co-treatment significantly inhibited EZH2 expression at the mRNA and protein level in EC-9706 and EC-109 cells (Fig. 5C and D).

MLT and 5-FU combination inhibits cell proliferation and promotes apoptosis by regulating EZH2 expression. The aforementioned results suggested that MLT and 5-FU combination significantly inhibited EZH2 expression. Thus, the present study hypothesized that MLT and 5-FU combination contributed to the chemotherapeutic effects of 5-FU by regulating EZH2 expression. To test this hypothesis, si-EZH2 or pcDNA-EZH2 were co-treated alongside MLT and 5-FU in EC-9706 and EC-109 cells. The results indicated that co-treatment of MLT, 5-FU and si-EZH2 synergistically inhibited EZH2 expression at the mRNA and protein level and further inhibited cell proliferation, invasion and migration, but promoted apoptosis of EC-9706 and EC-109 cells (Fig. 6A-F). In addition, co-treatment of MLT, 5-FU and si-EZH2 significantly inhibited the protein expression of Bcl-2 and Mcl-1, but enhanced the expression of cleaved caspase-3 (Fig. 6G). However, EZH2 overexpression significantly enhanced EZH2 expression at the mRNA and protein level, and reversed the effects of MLT and 5-FU on cell proliferation, invasion, migration, apoptosis and related protein expression in EC-9706 and EC-109 cells (Fig. 7A-H). These results suggested that MLT and 5-FU combination inhibited cell proliferation and promoted apoptosis by regulating EZH2 expression.

\section{Discussion}

China has been identified to have a relatively high incidence rate of EC, with $\sim 50 \%$ of new cases occurring each year. Although the incidence of EC has declined in recent years, the mortality rate remains fourth among malignant tumors, and the overall survival and 5-year survival rate of patients with EC remain low (30). Early diagnosis of EC is difficult due to its non-obvious clinical symptoms. The majority of patients with EC already present with locally advanced cancer or distant metastasis (3). Therefore, chemotherapy for the purpose of controlling the spread plays an important role in EC treatment. Although chemotherapeutic drugs for EC have made significant progress, 5-FU is still an important first-line chemotherapeutic drug (6). Therefore, it is urgent to improve the chemotherapeutic efficacy of 5-FU.

Recently, it was revealed that MLT exerts antitumor roles in studies of different types of human cancers, including pancreatic, liver, breast and colorectal cancer $(18,31,32)$. Studies have revealed that MLT can reduce the cell activity in bladder and colorectal cancer in a dose-dependent manner within a range of concentrations $(33,34)$. The present study determined that MLT could also reduce the cell activity of EC-9706 and EC-109 in a dose-dependent manner within a certain 

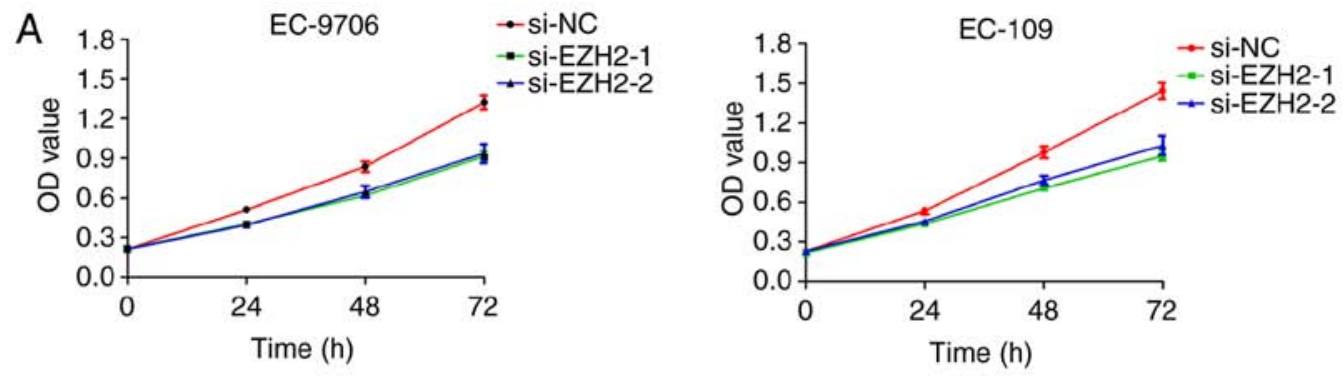

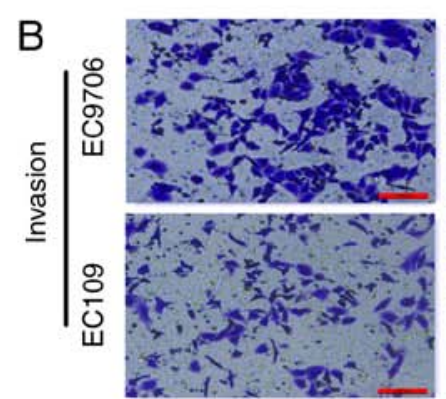

si-NC

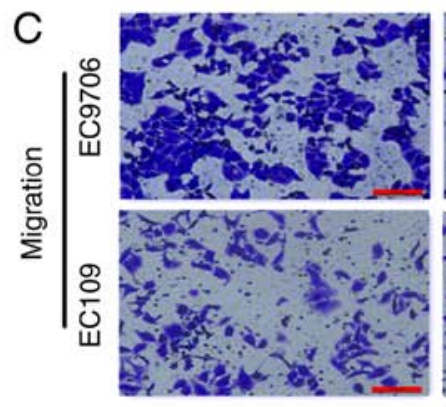

si-NC

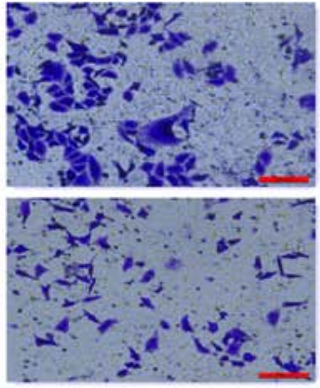

si-EZH2-1

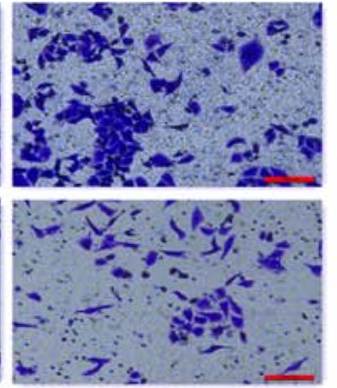

si-EZH2-2
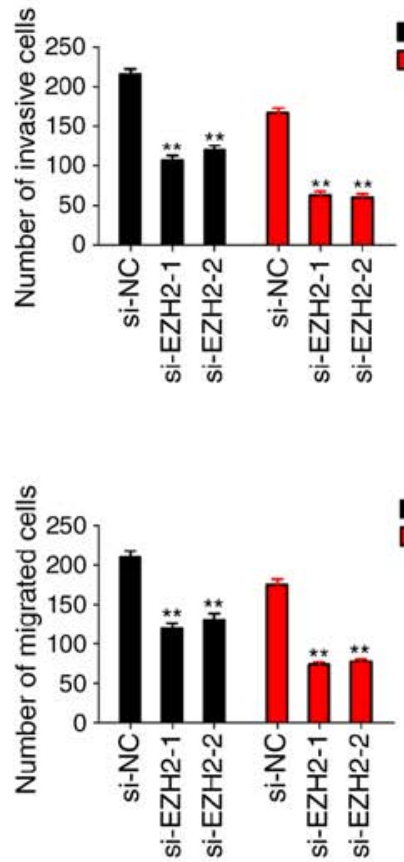

EC-9706

EC-109

EC-109

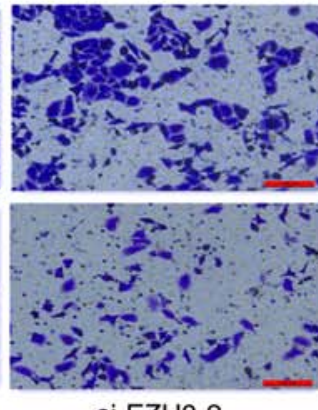

si-EZH2-2

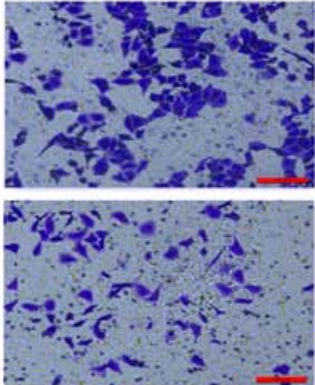

si-EZH2-1

si-EZH2-2
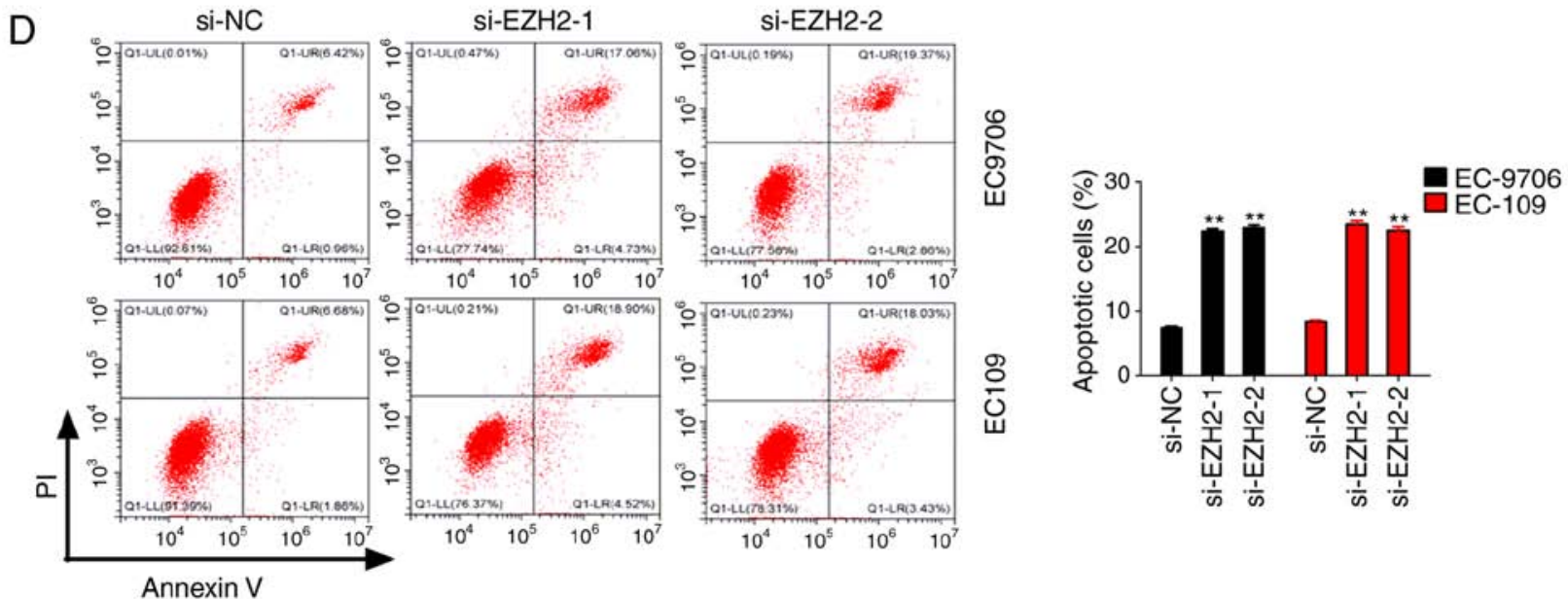

Figure 4. EZH2 acts as an oncogene in EC. (A) Effects of EZH2 knockdown on EC-9706 and EC-109 cell proliferation. (B and C) Effects of EZH2 knockdown on invasion and migration abilities in EC-9706 and EC-109 cells (scale bars, $100 \mu \mathrm{m}$ ). (D) Effects of EZH2 knockdown on EC-9706 and EC-109 cell apoptosis. ${ }^{* *} \mathrm{P}<0.01$ vs. the si-NC group. EZH2, histone-lysine N-methyltransferase EZH2; EC, esophageal cancer; si, small interfering RNA; NC, negative control.

concentration range. Recent studies have revealed that the combination of MLT and chemotherapeutic agents increased the efficacy of these agents. Xiang et al (17) revealed that the abnormal secretion of MLT inhibited aplasia Ras homology member I expression and mediated STAT3-induced paclitaxel resistance in breast cancer. Leja-Szpak et al (20) demonstrated that MLT and its metabolite enhanced gemcitabine chemosensitivity in pancreatic carcinoma cells. Hence, the present study assessed whether MLT combined with 5-FU can improve the chemotherapy sensitivity of 5-FU in EC.

To confirm the present hypothesis, cells were co-treated with 5-FU and MLT. MLT and 5-FU combination significantly enhanced 5-FU-mediated inhibition of cell activity and significantly decreased the $\mathrm{IC}_{50}$ of 5-FU in EC-9706 and EC-109 cells. Although no study has demonstrated that MLT increases 5-FU sensitivity in EC cells, a study 

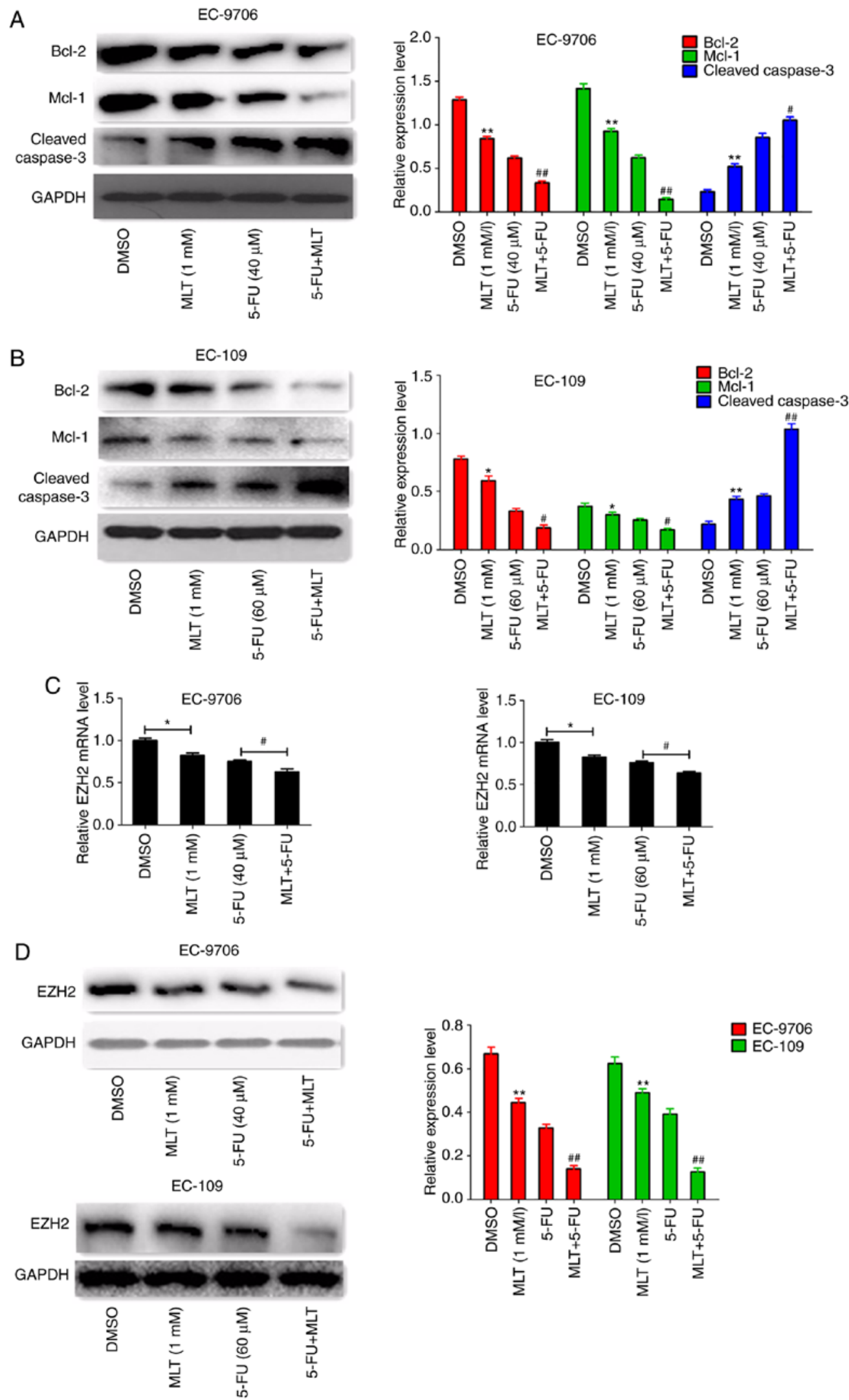

Figure 5. MLT and 5-FU combination suppresses EZH2 expression. (A and B) Effects of MLT and 5-FU combination on the protein expression of Bcl-2, Mcl-1 and cleaved caspase-3 in EC-9706 and EC-109 cells. (C and D) Effects of MLT and 5-FU combination on EZH2 expression at the mRNA and protein level in EC-9706 and EC-109 cells. ${ }^{*} \mathrm{P}<0.05$ and ${ }^{* *} \mathrm{P}<0.01$ vs. the DMSO group; ${ }^{\#} \mathrm{P}<0.05$ and ${ }^{\# \#} \mathrm{P}<0.01$ vs. the 5-FU group. EZH2, histone-lysine N-methyltransferase EZH2; MLT, melatonin; 5-FU, 5-fluorouracil.

has revealed that MLT enhanced 5-FU sensitivity and inhibited tumor cell growth in colorectal cancer cells (34).
Apoptosis or programmed cell death is a basic physiological process that plays a key role in cell development and tissue 


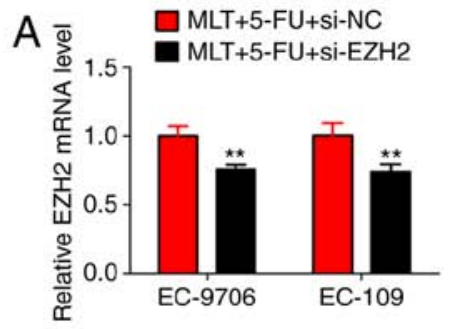

D
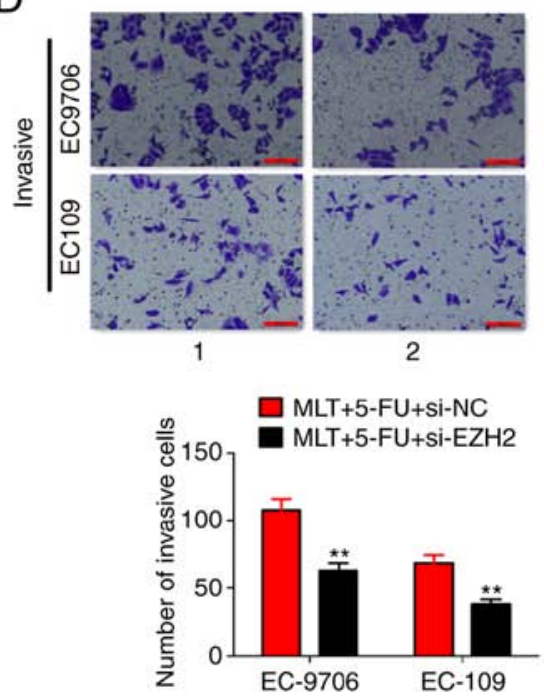

B $\quad \begin{aligned} & 1=M L T+5-F U+S i-N C \\ & 2=M L T+5-F U+S i-E Z H 2\end{aligned}$

EC-9706

EZH2

GAPDH

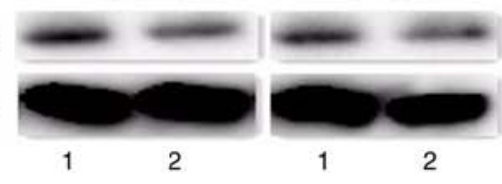

E
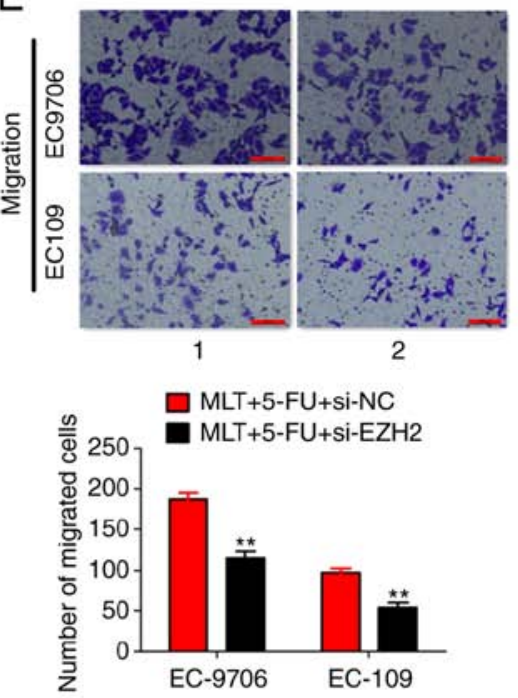

C $\square \mathrm{MLT}+5-\mathrm{FU}+\mathrm{Si}-\mathrm{NC}$

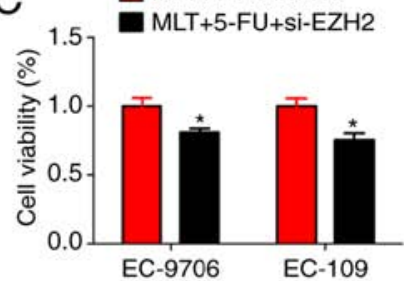

$\mathrm{F}$

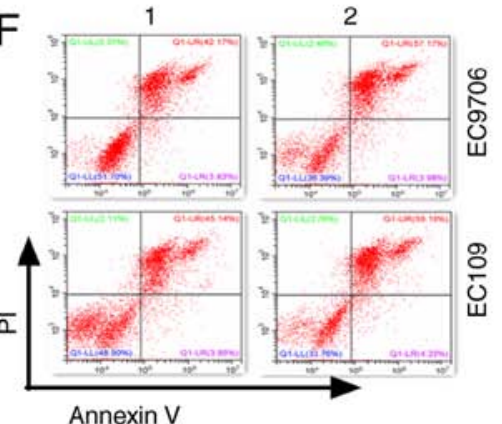

Annexin V
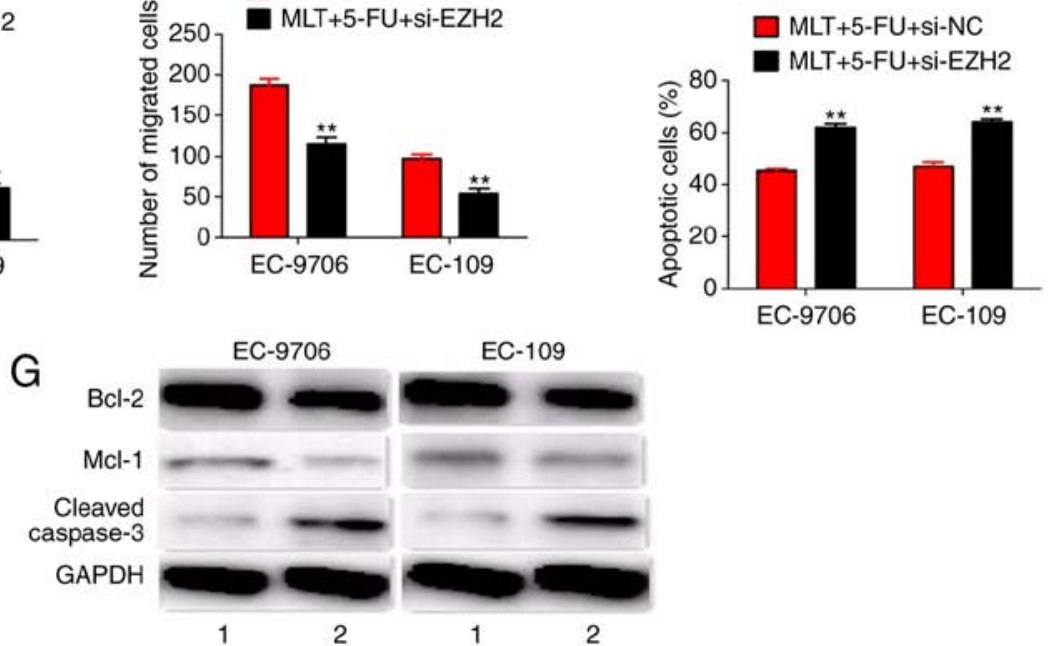

Figure 6. Co-treatment of MLT, 5-FU and si-EZH2. (A and B) Effects of MLT, 5-FU and si-EZH2 co-treatment on EZH2 expression. (C-F) Effects of MLT, 5-FU and si-EZH2 co-treatment on cell viability, invasion, migration and apoptosis (scale bars, $100 \mu \mathrm{m}$ ). (G) Effects of MLT, 5-FU and si-EZH2 co-treatment on protein expression. ${ }^{*} \mathrm{P}<0.05$ and ${ }^{* *} \mathrm{P}<0.01$ vs. MLT + 5-FU + si-NC group. EZH2, histone-lysine N-methyltransferase EZH2; MLT, melatonin; 5-FU, 5-fluorouracil; si, small interfering RNA; $\mathrm{NC}$, negative control.

homeostasis (35). Flow cytometric assays revealed that the effects of MLT and 5-FU on the sensitivity of EC cells was related to the induction of apoptosis. Bcl-2 and Mcl-1 are important apoptosis-regulating genes, while the expression of cleaved caspase- 3 directly indicates the level of apoptosis. The effects of MLT and 5-FU combination on the protein levels of Bcl-2, Mcl-1 and cleaved caspase-3 further confirmed its effect in promoting apoptosis.

The present results and TCGA database analysis revealed that EZH2 was upregulated in EC samples. Subsequently, EZH2 was confirmed as an oncogene in EC via biological experiments. Wang et al (23) revealed that EZH2 contributed to 5-FU resistance in gastric cancer by epigenetically suppressing F-box protein 32 expression. Rastgoo et al (24) determined that the EZH2/miR-138 axis contributed to drug resistance in multiple myeloma by downregulating RBPMS. In addition, previous studies also revealed that melatonin inhibited the tumorigenicity of glioblastoma stem-like cells via the EZH2 signaling axis $(25,26)$. Hence, the present study hypothesized that the MLT and 5-FU combination inhibited cell proliferation and promoted apoptosis by regulating EZH2 expression. First, the present study confirmed that MLT and 5-FU significantly inhibited EZH2 expression via RT-qPCR and western blotting assays. Next, the present study confirmed that MLT and 5-FU combination improved the sensitivity of 5-FU to EC cells by downregulating EZH2 expression through co-treatment experiments. As an important oncogene, EZH2 may affect the malignancy of tumors through multiple pathways. In a previous study it was revealed that melatonin inhibited glioblastoma stem-like cells via the EZH2-NOTCH1 signaling axis (25). Another study revealed that melatonin inhibited glioblastoma stem-like cells via AKT-EZH2-STAT3 signaling axis (26). One of our ongoing studies also revealed that EZH2 effected the development of ESCC by activating the JKA2/STAT3 signaling pathway. Hence, it was hypothesized that the effects of melatonin and 5-FU combination on the malignancy of esophageal cancer may have been achieved by 

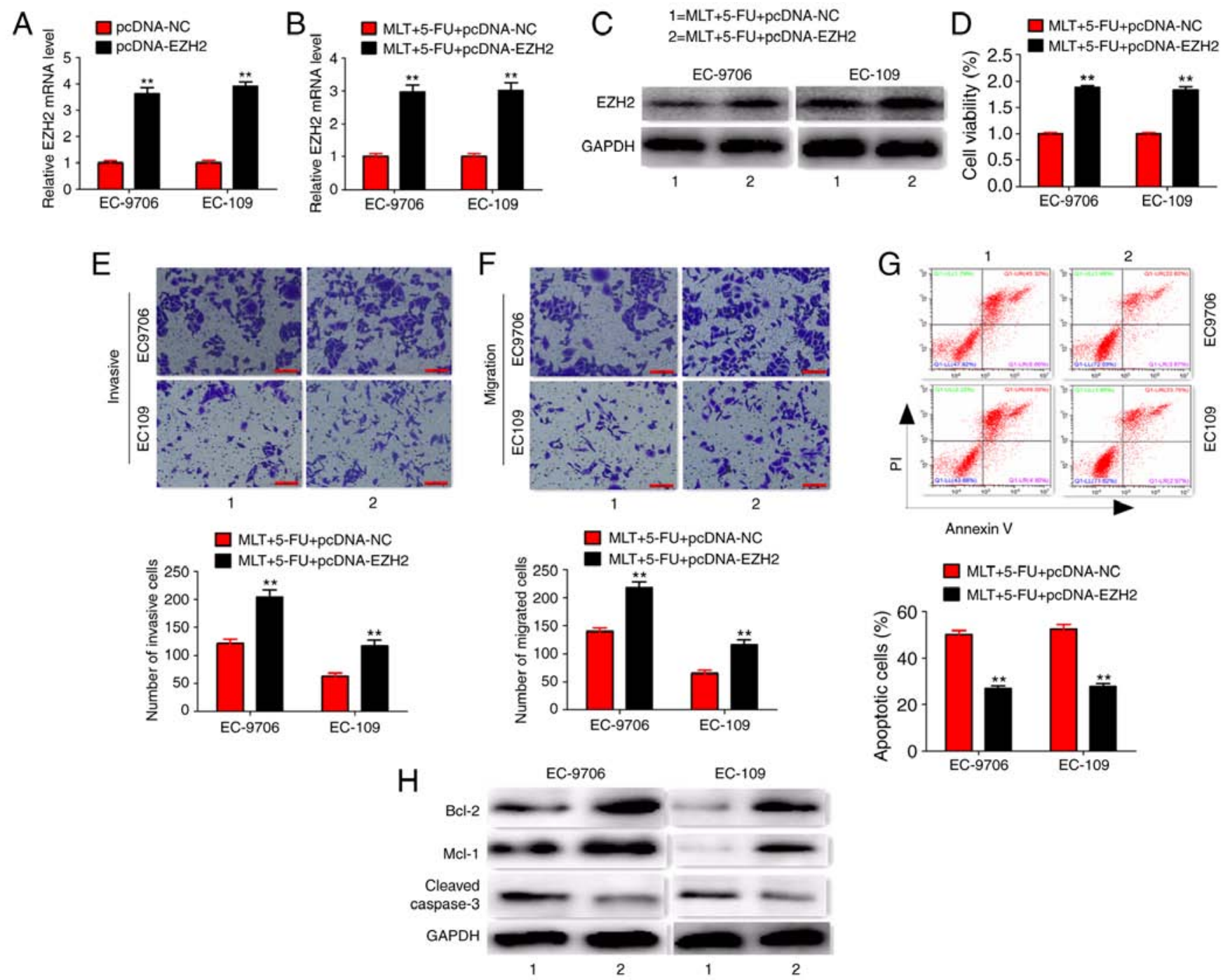

Figure 7. Co-treatment of MLT, 5-FU and pcDNA-EZH2. (A) Effects of pcDNA-EZH2 treatment on EZH2 expression (B and C) Effects of MLT, 5-FU and pcDNA-EZH2 co-treatment on EZH2 expression. (D-G) Effects of MLT, 5-FU and pcDNA-EZH2 co-treatment on cell viability, invasion, migration and apoptosis (scale bars, $100 \mu \mathrm{m}$ ). (H) Effects of MLT, 5-FU and pcDNA-EZH2 co-treatment on protein expression. ${ }^{* *} \mathrm{P}<0.01 \mathrm{vs}$. MLT + 5-FU + pcDNA-NC group. EZH2, histone-lysine N-methyltransferase EZH2; MLT, melatonin; 5-FU, 5-fluorouracil; NC, negative control.

regulating the EZH2/JKA2/STAT3 signaling pathway. However, this hypothesis requires further experiments for confirmation.

In conclusion, the present experimental approach demonstrated that MLT enhanced 5-FU-mediated inhibition of cell proliferation via the promotion of apoptosis by regulating EZH2 expression in EC cells. This combination treatment may potentially be a more effective treatment option in EC chemotherapy.

\section{Acknowledgements}

Not applicable.

\section{Funding}

The present study was supported by the Medical Science Research Project of Henan Province (grant no. LHGJ20191039); Science and Technology Project of Henan Province (grant no. 212102310121).

\section{Availability of data and materials}

The datasets used and/or analyzed during the present study are available from the corresponding author on reasonable request.

\section{Authors' contributions}

MengtZ, MenglZ and RL performed the majority of the experiments in the study. YZ and RZ contributed to the analysis of the experimental data. MengtZ and $\mathrm{YZ}$ contributed to the study design, manuscript writing and provided experimental funding support. All authors read and approved the manuscript and agree to be accountable for all aspects of the research in ensuring that the accuracy or integrity of any part of the work are appropriately investigated and resolved.

\section{Ethics approval and consent to participate}

Not applicable. 


\section{Patient consent for publication}

Not applicable.

\section{Competing interests}

The authors declare that they have no competing interests.

\section{References}

1. Pennathur A, Gibson MK, Jobe BA and Luketich JD: Oesophageal carcinoma. Lancet 381: 400-412, 2013.

2. Ferlay J, Soerjomataram I, Dikshit R, Eser S, Mathers C, Rebelo M, Parkin DM, Forman D and Bray F: Cancer incidence and mortality worldwide: Sources, methods and major patterns in GLOBOCAN 2012. Int J Cancer 136: E359-E386, 2015.

3. Tu CC and Hsu PK: The frontline of esophageal cancer treatment: Questions to be asked and answered. Ann Transl Med 6: 83, 2018

4. Kimura M, Ishiguro $H$, Tanaka $T$ and Takeyama $H$ : Advanced esophageal cancer with tracheobronchial fistula successfully treated by esophageal bypass surgery. Int J Surg Case Rep 9: $115-118,2015$

5. Li B, Hong P, Zheng CC, Dai W, Chen WY, Dai W, Chen WY, Yang QS, Han L, Tsao SW, et al: Identification of miR-29c and its target FBXO31 as a key regulatory mechanism in esophageal cancer chemoresistance: Functional validation and clinical significance. Theranostics 9: 1599-1613, 2019.

6. Siersema PD: Esophageal cancer awareness issue 2019. Endoscopy 51: 291-292, 2019.

7. Zhang N, Yin Y, Xu SJ and Chen WS: 5-Fluorouracil: Mechanisms of resistance and reversal strategies. Molecules 13: 1551-1569, 2008.

8. Vodenkova S, Buchler T, Cervena K, Veskrnova V, Vodicka P and Vymetalkova V: 5-Fluorouracil and other fluoropyrimidines in colorectal cancer: Past, present and future. Pharmacol Ther 206: $107447,2020$.

9. Acuna-Castroviejo D, Escames G, Venegas C, Diaz-Casado ME, Lima-Cabello E, López LC, Rosales-Corral S, Tan DX and Reiter RJ: Extrapineal melatonin: Sources, regulation, and potential functions. Cell Mol Life Sci 71: 2997-3025, 2014.

10. Claustrat B and Leston J: Melatonin: Physiological effects in humans. Neurochirurgie 61: 77-84, 2015.

11. Talib WH: Melatonin and cancer hallmarks. Molecules 23: 518, 2018.

12. Cardinali DP: Melatonin: Clinical perspectives in neurodegeneration. Front Endocrinol (Lausanne) 10: 480, 2019.

13. Zhang C, Yang XZ, Xu MJ, Huang GY, Zhang Q, Cheng YX, He L and Ren HY: Melatonin promotes cheliped regeneration, digestive enzyme function, and immunity following autotomy in the Chinese mitten crab, eriocheir sinensis. Front Physiol 9: 269, 2018

14. Zhou N, Wei ZX and Qi ZX: Inhibition of autophagy triggers melatonin-induced apoptosis in glioblastoma cells. BMC Neurosci 20: 63, 2019.

15. Xu K, Wang J, Liu H, Zhao J and Lu W: Melatonin promotes the proliferation of chicken sertoli cells by activating the ERK/Inhibin alpha subunit signaling pathway. Molecules 25: 1230, 2020.

16. Song J, Ma SJ,Luo JH,Zhang H, Wang RX, Liu H, Li L, Zhang ZG and Zhou RX: Melatonin induces the apoptosis and inhibits the proliferation of human gastric cancer cells via blockade of the AKT/MDM2 pathway. Oncol Rep 39: 1975-1983, 2018.

17. Xiang S, Dauchy RT, Hoffman AE, Pointer D, Frasch T, Blask DE and Hill SM: Epigenetic inhibition of the tumor suppressor ARHI by light at night-induced circadian melatonin disruption mediates STAT3-driven paclitaxel resistance in breast cancer. J Pineal Res 67: e12586, 2019.

18. Wang Q, Sun Z, Du L, Xu C, Wang Y, Yang B, He N, Wang J, Ji K, Liu Y and Liu Q: Melatonin sensitizes human colorectal cancer cells to $\gamma$-ray ionizing radiation in vitro and in vivo. Int $\mathrm{J}$ Mol Sci 19: 3974, 2018
19. Hao J, Fan W, Li Y, Tang R, Tian C, Yang Q, Zhu T, Diao C, Hu S, Chen M, et al: Melatonin synergizes BRAF-targeting agent vemurafenib in melanoma treatment by inhibiting iNOS/hTERT signaling and cancer-stem cell traits. J Exp Clin Cancer Res 38: 48, 2019.

20. Leja-Szpak A, Nawrot-Porąbka K, Góralska M, Jastrzębska M, Link-LenczowskiP,BoniorJ,PierzchalskiPand JaworekJ:Melatonin and its metabolite N1-acetyl-N2-formyl-5-methoxykynuramine (afmk) enhance chemosensitivity to gemcitabine in pancreatic carcinoma cells (PANC-1). Pharmacol Rep 70: 1079-1088, 2018.

21. Bae WK and Hennighausen L: Canonical and non-canonical roles of the histone methyltransferase EZH2 in mammary development and cancer. Mol Cell Endocrinol 382: 593-597, 2014.

22. Wang J, Yang X, Li R, Zhang R, Hu D, Zhang Y and Gao L: LncRNA SNHG6 inhibits apoptosis by regulating EZH2 expression via the sponging of MiR-101-3p in esophageal squamous-cell carcinoma. Onco Targets Ther 13: 11411-11420, 2020.

23. Wang C, Li X, Zhang J, Ge Z, Chen $\mathrm{H}$ and Hu J: EZH2 contributes to 5-FU resistance in gastric cancer by epigenetically suppressing FBXO32 expression. Onco Targets Ther 11: 7853-7864, 2018.

24. Rastgoo N, Pourabdollah M, Abdi J, Reece D and Chang H: Dysregulation of EZH2/miR-138 axis contributes to drug resistance in multiple myeloma by downregulating RBPMS. Leukemia 32: 2471-2482, 2018.

25. Zheng X, Pang B, Gu G, Gao T, Zhang R, Pang Q and Liu Q Melatonin inhibits glioblastoma stem-like cells through suppression of EZH2-NOTCH1 signaling axis. Int J Biol Sci 13: 245-253, 2017.

26. Chen X, Hao A, Li X, Du Z, Li H, Wang H, Yang H and Fang Z: Melatonin inhibits tumorigenicity of glioblastoma stem-like cells via the AKT-EZH2-STAT3 signaling axis. J Pineal Res 61: 208-217, 2016

27. Li Z, Jiang C and Yuan Y: TCGA based integrated genomic analyses of ceRNA network and novel subtypes revealing potential biomarkers for the prognosis and target therapy of tongue squamous cell carcinoma. PLoS One 14: e0216834, 2019.

28. Livak KJ and Schmittgen TD: Analysis of relative gene expression data using real-time quantitative PCR and the 2(-Delta Delta C(T)) method. Methods 25: 402-408, 2001.

29. Zhang Y, Li R, Ding X, Zhang K and Qin W: Upregulation of long non-coding RNA SNHG6 promote esophageal squamous cell carcinoma cell malignancy and its diagnostic value. Am J Transl Res 11: 1084-1091, 2019.

30. Bray F, Ferlay J, Soerjomataram I, Siegel RL, Torre LA and Jemal A: Global cancer statistics 2018: GLOBOCAN estimates of incidence and mortality worldwide for 36 cancers in 185 countries. CA Cancer J Clin 68: 394-424, 2018.

31. Reiter RJ, Rosales-Corral SA, Tan DX, Acuna-Castroviejo D, Qin L, Yang SF and Xu K: Melatonin, a full service anti-cancer agent: Inhibition of initiation, progression and metastasis. Int J Mol Sci 18: 843, 2017.

32. Hill SM, Belancio VP, Dauchy RT, Xiang S, Brimer S, Mao L, Hauch A, Lundberg PW, Summers W, Yuan L, et al: Melatonin: An inhibitor of breast cancer. Endocr Relat Cancer 22: R183-R204, 2015.

33. Chen YT, Yang CC, Shao PL, Huang CR and Yip HK: Melatonin-mediated downregulation of ZNF746 suppresses bladder tumorigenesis mainly through inhibiting the AKT-MMP-9 signaling pathway. J Pineal Res 66: e12536, 2019.

34. Gao Y, Xiao X, Zhang C, Yu W, Guo W, Zhang Z, Li Z, Feng X, Hao J, Zhang K, et al: Melatonin synergizes the chemotherapeutic effect of 5-fluorouracil in colon cancer by suppressing PI3K/AKT and NF- $\kappa \mathrm{B} / \mathrm{iNOS}$ signaling pathways. J Pineal Res 62: e12380, 2017.

35. D'Arcy MS: Cell death: A review of the major forms of apoptosis, necrosis and autophagy. Cell Biol Int 43: 582-592, 2019.

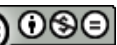

This work is licensed under a Creative Commons Attribution-NonCommercial-NoDerivatives 4.0 International (CC BY-NC-ND 4.0) License. 